Nonlinear Processes in Geophysics (2005) 12: 353-361

SRef-ID: $1607-7946 / \mathrm{npg} / 2005-12-353$

European Geosciences Union

(C) 2005 Author(s). This work is licensed

under a Creative Commons License.

\title{
Fractal behaviour of the seismicity in the Southern Iberian Peninsula
}

\author{
X. Lana ${ }^{1}$, M. D. Martínez ${ }^{2}$, A. M. Posadas ${ }^{3,4}$, and J. A. Canas ${ }^{5}$ \\ ${ }^{1}$ Departament de Física i Enginyeria Nuclear, Univ. Politècnica de Catalunya, Av. Diagonal 647, 08028 Barcelona, Spain \\ ${ }^{2}$ Departament de Física Aplicada, Universitat Politècnica de Catalunya, Av. Diagonal 649, 08028 Barcelona, Spain \\ ${ }^{3}$ Departamento de Física Aplicada, Universidad de Almería, 04120 Almería, Spain \\ ${ }^{4}$ Instituto Andaluz de Geofísica y de Prevención de Desastres Sísmicos, Universidad de Granada, 18080 Granada, Spain \\ ${ }^{5}$ Departament d'Enginyeria del Terreny, Geofísica i Cartográfica, Universitat Politècnica de Catalunya, Gran Capità s/n, \\ 08034 Barcelona, Spain
}

Received: 12 July 2004 - Revised: 11 February 2005 - Accepted: 12 February 2005 - Published: 25 February 2005

Part of Special Issue "Seismicity pattern dynamics"

\begin{abstract}
The fractal behaviour of the seismicity in the Southern Iberian Peninsula is analysed by considering two different series of data: the distance and the elapsed time between consecutive seismic events recorded by the seismic network of the Andalusian Institute of Geophysics (AIG). The fractal analyses have been repeated by considering four threshold magnitudes of 2.5, 3.0, 3.5 and 4.0. The re-scaled analysis lets to determine if the seismicity shows strong randomness or if it is characterised by time-persistence and the cluster dimension indicates the degree of time and spatial clustering of the seismicity. Another analysis, based on the reconstruction theorem, permits to evaluate the minimum number of nonlinear equations describing the dynamical mechanism of the seismicity, its "loss of memory", its chaotic character and the instability of a possible predicting algorithm. The results obtained depict some differences depending on distances or elapsed times and the different threshold levels of magnitude also lead to slightly different results. Additionally, only a part of the fractal tools, the rescaled analysis, have been applied to five seismic crises in the same area.
\end{abstract}

\section{Introduction}

The most relevant patterns of a complex nonlinear and dissipative dynamical system, as that of the seismic activity, can be determined by means of fractal theory. Some examples can be found in Smalley et al. (1987), who analysed earthquake clustering, Correig and Urquizú (1996), who studied the chaotic behaviour of Coda waves, Correig et

Correspondence to: X. Lana

(francisco.javier.lana@upc.edu) al. (1997a, b), who characterised the temporal occurrence of events and studied the self-organised criticality of aftershocks, and Urquizú and Correig (1998), who analysed several types of seismic signals. It has to be also mentioned the book of Goltz (1997), where the fractal and chaotic properties of earthquakes are extensively analysed.

The elapsed times and distances between consecutive seismic events belonging to the 1985-2000 Southern Iberian Peninsula activity (Fig. 1a) are analysed by taking as database the seismic catalogue of the Andalusian Institute of Geophysics (IAG), which compiles more than 20000 events. With the aim of establishing the possible role of the seismic magnitude, computations have been repeated for four different threshold magnitudes of 2.5, 3.0, 3.5 and 4.0. The Gutenberg-Richter law for this catalogue suggests that events with magnitude less than 2.5 are not always well-detected and therefore lower threshold magnitudes have not been considered. Additionally, if we want to obtain results with low uncertainties, it is not advisable to apply fractal techniques to series of distances and elapsed times of short length. Nevertheless, the re-scaled analysis has been successfully used to obtain the Hurst exponent for five seismic crises in the Southern Iberian Peninsula, with the aim of detecting possible differences in the behaviour of the seismic crises and the whole seismic catalogue. A detailed description of these five crises can be found in Posadas et al. (2002). Completeness of these sets of aftershocks is assured by the fulfilment of the Omori's law and by choosing magnitude intervals for which the Gutemberg-Richter law is well fitted. Unfortunately, other fractal techniques, as the cluster analysis and analyses based on the reconstruction theorem need for longer series of data and only the whole catalogue has been analysed. 
Table 1. Some characteristics of the complete seismic catalogue and 5 seismic crisis, from which the series of distances and elapsed times between consecutive events are derived.

\begin{tabular}{cccccc}
\hline & $\mathrm{m}_{\mathrm{b}}$ range & $\mathrm{n}_{0}$, events & Recording time & $\theta$ (latitude) & $\varphi$ (longitude) \\
\hline & $\geq 2.5$ & 5648 & $1985-2000$ & $35^{\circ} \mathrm{N}-40^{\circ} \mathrm{N}$ & $-12^{\circ} \mathrm{W}-0^{\circ} \mathrm{E}$ \\
IAG & $\geq 3.0$ & 1551 & $1985-2000$ & $35^{\circ} \mathrm{N}-40^{\circ} \mathrm{N}$ & $-12^{\circ} \mathrm{W}-0^{\circ} \mathrm{E}$ \\
catalogue & $\geq 3.5$ & 441 & $1985-2000$ & $35^{\circ} \mathrm{N}-40^{\circ} \mathrm{N}$ & $-12^{\circ} \mathrm{W}-0^{\circ} \mathrm{E}$ \\
& $\geq 4.0$ & 103 & $1985-2000$ & $35^{\circ} \mathrm{N}-40^{\circ} \mathrm{N}$ & $-12^{\circ} \mathrm{W}-0^{\circ} \mathrm{E}$ \\
\hline Adra & $\geq 1.5$ & 346 & Dec.1993-Mar. 1994 & $36.4^{\circ} \mathrm{N}-37.0^{\circ} \mathrm{N}$ & $2.5^{\circ} \mathrm{W}-3.3^{\circ} \mathrm{W}$ \\
crisis & $(\geq 2.0)$ & $(764)$ & $1985-2000$ & $36.4^{\circ} \mathrm{N}-37.0^{\circ} \mathrm{N}$ & $2.5^{\circ} \mathrm{W}-3.3^{\circ} \mathrm{W}$ \\
\hline Agrón & $\geq 1.5$ & 101 & $5-15$ Dec. 1988 & $36.99^{\circ} \mathrm{N}-37.05^{\circ} \mathrm{N}$ & $3.8^{\circ} \mathrm{W}-3.9^{\circ} \mathrm{W}$ \\
crisis & $(\geq 1.5)$ & $(525)$ & $1985-2000$ & $36.99^{\circ} \mathrm{N}-37.05^{\circ} \mathrm{N}$ & $3.8^{\circ} \mathrm{W}-3.9^{\circ} \mathrm{W}$ \\
\hline Alborán & $\geq 2.5$ & 56 & Jun.1997-Jan. 1998 & $36.3^{\circ} \mathrm{N}-36.7^{\circ} \mathrm{N}$ & $2.8^{\circ} \mathrm{W}-3.4^{\circ} \mathrm{W}$ \\
crisis & $(\geq 2.0)$ & $(523)$ & $1985-2000$ & $36.3^{\circ} \mathrm{N}-36.7^{\circ} \mathrm{N}$ & $2.8^{\circ} \mathrm{W}-3.4^{\circ} \mathrm{W}$ \\
\hline Antequera & $\geq 2.5$ & 79 & $7-9$ Jun.1989 & $36.9^{\circ} \mathrm{N}-37.3^{\circ} \mathrm{N}$ & $4.4^{\circ} \mathrm{W}-4.8^{\circ} \mathrm{W}$ \\
crisis & $(\geq 2.0)$ & $(288)$ & $1985-2000$ & $36.9^{\circ} \mathrm{N}-37.3^{\circ} \mathrm{N}$ & $4.4^{\circ} \mathrm{W}-4.8^{\circ} \mathrm{W}$ \\
\hline Loja & $\geq 2.0$ & 146 & Feb. -Jun. 1985 & $37.1^{\circ} \mathrm{N}-37.2^{\circ} \mathrm{N}$ & $4.1^{\circ} \mathrm{W}-4.3^{\circ} \mathrm{W}$ \\
crisis & $(\geq 2.0)$ & $(950)$ & $1985-2000$ & $37.1^{\circ} \mathrm{N}-37.2^{\circ} \mathrm{N}$ & $4.1^{\circ} \mathrm{W}-4.3^{\circ} \mathrm{W}$ \\
\hline
\end{tabular}

For a better understanding of the database and an easier discussion of the results, Table 1 includes some characteristics of the different datasets employed. $m_{b}$ designs the range of body-wave magnitude, $n_{0}$ the number of events (number of elements of the series to be analysed), $t_{0}$ dates of the seismic episode and $\theta$ and $\varphi$ the range of latitude and longitude of the area where the seismic crisis was detected. Cases within parenthesis correspond to sets of seismic events located within the area defined by a seismic crisis, but extending in time to the 1985-2000 recording period. The well detected minimum magnitude according to the GutembergRichter law is below 2.5 for some crisis due to the close monitoring by the IAG of these areas.

\section{Fractal techniques}

A first approach to the fractal behaviour of the series consists of the cluster analysis (Smalley et al., 1987; Korvin, 1992; Posadas et al., 2002). The series of distances or elapsed times between consecutive events are divided in different subsets, each of them with the same number $n$ of elements. $P\left(n, C_{0}\right)$ is defined as the probability of detecting in a subset of $n$ elements a distance or elapsed time exceeding a threshold value $C_{0}$, which will be given in kilometres or days depending on the series analysed. If the series have a fractal behaviour, $P\left(n, C_{0}\right)$ will depend on $n$ according to the potential law

$$
\log \left[P\left(n, C_{0}\right)\right]=a \log [n]+b
$$

and the cluster dimension $D$ will be equal to $1-a$. It should be remembered that the fulfilment of the potential law (Eq. 1) by the empiric data is a sign of fractal behaviour.

The re-scaled analysis and the Hurst exponent provide us with another interesting point of view of the behaviour of complex dynamic systems (Feder, 1988). Some examples of this fractal technique, applied to different fields in geology and geophysics, can be found in Korvin (1992) and Turcotte (1992), among others. Very briefly, this process consists of the evaluation of mean values, cumulative differences, maximum ranges $R(n)$ of the integrated signal and standard deviations $S(n)$ for subsets of series with different number $n$ of elements. If the fractal behaviour is confirmed, with $H$ being the Hurst exponent

$$
\frac{R(n)}{S(n)}=c n^{H} \text {. }
$$

Values of $H$ close to 0.5 indicate randomness of the analysed series, while values of $H$ clearly exceeding 0.5 indicate persistence in the dynamical mechanism governing the seismic activity.

One of the most complete descriptions of a complex dynamical system is achieved by means of a fractal analysis based on the reconstruction theorem (Takens, 1981; Grassberger and Procaccia, 1983a, b). This analysis implies the computation of the correlation dimension $\mu$, the Kolmogorov entropy $K$ and the Lyapunov exponents $\lambda$.

The space of the dynamical system is reconstructed by means of m-dimension vectors

$Z_{i}=\{x(i), x(i+q), \ldots, x[i+q(m-1)]\}$,

which are generated from the series $x(i)$ of elapsed times or distances. The integer number $q$ is a shift parameter, which is chosen equal to the lag order for which auto-correlation function of the series analysed achieves its first null value. Alternative ways of choosing $q$ will be introduced in the introduction of the results. The correlation dimension and the Kolmogorov entropy are derived from the correlation integral (Diks, 1999)

$$
C(r)=\lim _{N \rightarrow \infty}\left\{\frac{1}{N^{2}} \sum_{i, j=1}^{N} H\left[r-\left|Z_{i}-Z_{j}\right|\right]\right\}
$$




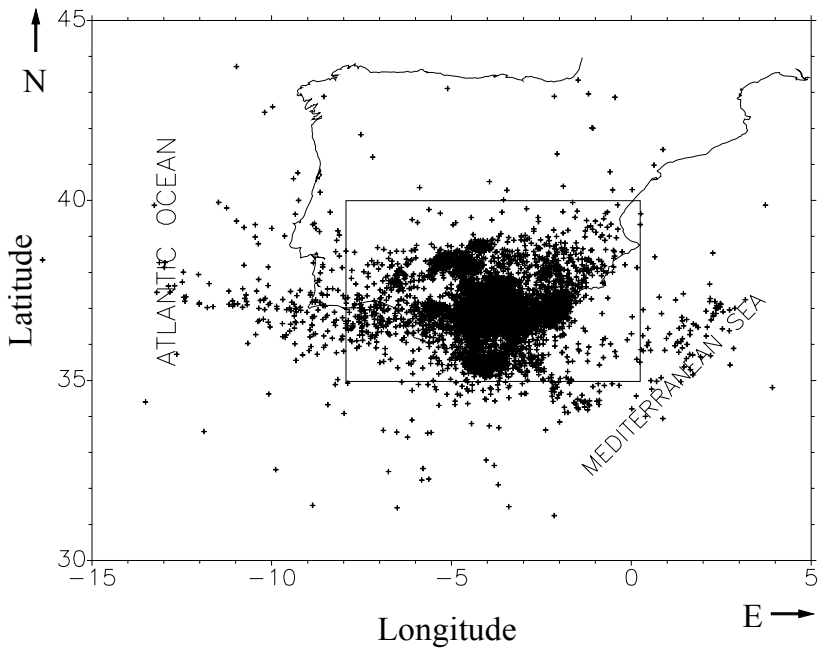

Fig. 1. Location of the area analysed (inner rectangle) with the spatial distribution of seismic events recorded by the Andalusian Institute of Geophysics.

$H(\bullet)$ represents the Heaviside function and $\boldsymbol{Z}_{\boldsymbol{i}}$ and $\boldsymbol{Z}_{\boldsymbol{j}}$ are pairs of vectors of the space of the dynamical system. This equation can be interpreted as the number of points of the dynamical system inside a sphere of radius $r$. The euclidean metrics is assumed to evaluate the distance between pairs of vectors $\left(\boldsymbol{Z}_{\boldsymbol{i}}, \boldsymbol{Z}_{\boldsymbol{j}}\right)$. After the definition of the correlation integral, it is straightforward to introduce the correlation dimension

$\mu=\lim _{r \rightarrow 0} \frac{\log [C(r)]}{\log [r]}$

which constitutes a measure of the minimum number of nonlinear equations describing the dynamic system. In fact, a stationary value of $\mu$ is assumed to be a very good approach to the correlation dimension for a high enough dimension $m$, usually designed as embedding dimension $d_{E}$. A high embedding dimension is a sign of random behaviour of the dynamical system. Additionally, bearing in mind Eq. (4), the Kolmogorov entropy (Cohen and Procaccia, 1983) is defined as

$K=\frac{1}{q} \lim _{m \rightarrow \infty} \lim _{r \rightarrow 0}\left[\ln \left\{\frac{C_{m}(r)}{C_{m+1}(r)}\right\}\right]$,

which is a consequence of the asymptotic evolution of $C(r)$ (Diks, 1999)

$C_{m}(r) \approx r^{\mu} e^{-q m K}$

$K$ is a definite positive parameter that measures the "loss of memory" of the dynamical mechanism and characterises its chaotic behaviour. $K$ equal to zero would indicate a null "loss of memory" and an absolutely deterministic mechanism. On the contrary, a high value of the Kolmogorov entropy is a sign of chaotic behaviour and relevant "loss of memory", which are outstanding shortcomings for a successful forecasting of future states of the dynamical system. A
Table 2. Evolution of the cluster dimension, $D$, with the distance and the elapsed time for different threshold levels $C_{0}$ given in $\mathrm{km}$ and days. The square regression coefficients for the linear segments in the log-log scale are included within parenthesis.

\begin{tabular}{|c|c|c|c|}
\hline \multicolumn{2}{|r|}{$m_{b}=2.5$} & \multicolumn{2}{|r|}{$m_{b}=3.0$} \\
\hline$C_{0}(\mathrm{~km})$ & $D$ & $C_{0}(\mathrm{~km})$ & $D$ \\
\hline 50 & $0.803(0.978)$ & 50 & $0.774(0.999)$ \\
\hline 100 & $0.699(0.987)$ & 100 & $0.670(0.999)$ \\
\hline 150 & $0.593(0.993)$ & 150 & $0.581(0.998)$ \\
\hline 200 & $0.484(0.996)$ & 200 & $0.482(0.992)$ \\
\hline 250 & $0.370(0.999)$ & 250 & $0.333(0.995)$ \\
\hline 300 & $0.264(0.999)$ & 300 & $0.271(0.997)$ \\
\hline 350 & $0.215(0.998)$ & 350 & $0.179(0.998)$ \\
\hline 400 & $0.115(0.999)$ & 400 & $0.150(0.998)$ \\
\hline 450 & $0.058(0.999)$ & 450 & $0.120(0.997)$ \\
\hline 500 & $0.042(0.999)$ & 500 & $0.090(0.997)$ \\
\hline 550 & $0.023(0.999)$ & 550 & $0.075(0.995)$ \\
\hline \multicolumn{2}{|r|}{$m_{b}=2.5$} & \multicolumn{2}{|r|}{$m_{b}=3.0$} \\
\hline$C_{0}$ (days) & $D$ & $C_{0}$ (days) & $D$ \\
\hline 0.5 & $0.555(0.984)$ & 1.0 & $0.688(0.979)$ \\
\hline 1.0 & $0.396(0.999)$ & 2.0 & $0.551(0.985)$ \\
\hline 1.5 & $0.292(0.999)$ & 3.0 & $0.443(0.991)$ \\
\hline 2.0 & $0.223(0.998)$ & 4.0 & $0.351(0.985)$ \\
\hline 2.5 & $0.202(0.997)$ & 5.0 & 0.304 (0.989) \\
\hline 3.0 & $0.155(0.997)$ & 6.0 & $0.264(0.993)$ \\
\hline 3.5 & $0.169(0.998)$ & 7.0 & $0.224(0.989)$ \\
\hline 4.0 & $0.136(0.998)$ & 8.0 & $0.215(0.988)$ \\
\hline 4.5 & $0.132(0.997)$ & 9.0 & $0.180(0.992)$ \\
\hline 5.0 & $0.108(0.998)$ & 10.0 & $0.147(0.922)$ \\
\hline 5.5 & $0.106(0.997)$ & 11.0 & $0.143(0.995)$ \\
\hline 6.0 & $0.102(0.998)$ & 12.0 & $0.110(0.995)$ \\
\hline 6.5 & $0.093(0.998)$ & 13.0 & $0.122(0.997)$ \\
\hline 7.0 & $0.081(0.998)$ & 14.0 & $0.105(0.997)$ \\
\hline 7.5 & $0.043(0.997)$ & 15.0 & $0.088(0.995)$ \\
\hline 8.0 & $0.040(0.998)$ & 16.0 & $0.103(0.996)$ \\
\hline
\end{tabular}

complete description of shortcomings that could appear in the computation of the correlation dimension and the entropy are described in Theiler (1988, 1990) and Diks (1999).

The last relevant feature of this fractal analysis are the Lyapunov exponents, which are a measure of the instability of the dynamical system. Very briefly, the largest Lyapunov exponent $\lambda$ can be introduced as

$\lambda=\lim _{i \rightarrow \infty}\left\{\frac{1}{i} \ln \left\|\frac{\delta Z_{i}}{\delta Z_{0}}\right\|\right\}$

with $\|\bullet\|$ being the norm of the Jacobian matrix, $\delta Z_{0}$ an infinitesimal change in the starting point of the space of the dynamical system and $\delta Z_{i}$ the corresponding change for the ith vector, reconstructed according to Eq. (3). If $\lambda$ is positive, a future state of the dynamical system (the long-term prediction) could strongly depend on uncertainties or perturbations on the initial state, and unstable predictability will characterise the process. A detailed description of a computational algorithm to obtain not only the largest Lyapunov exponent, but all the Lyapunov exponents $\lambda_{j}\left(j=1, \ldots, d_{E}\right)$ 


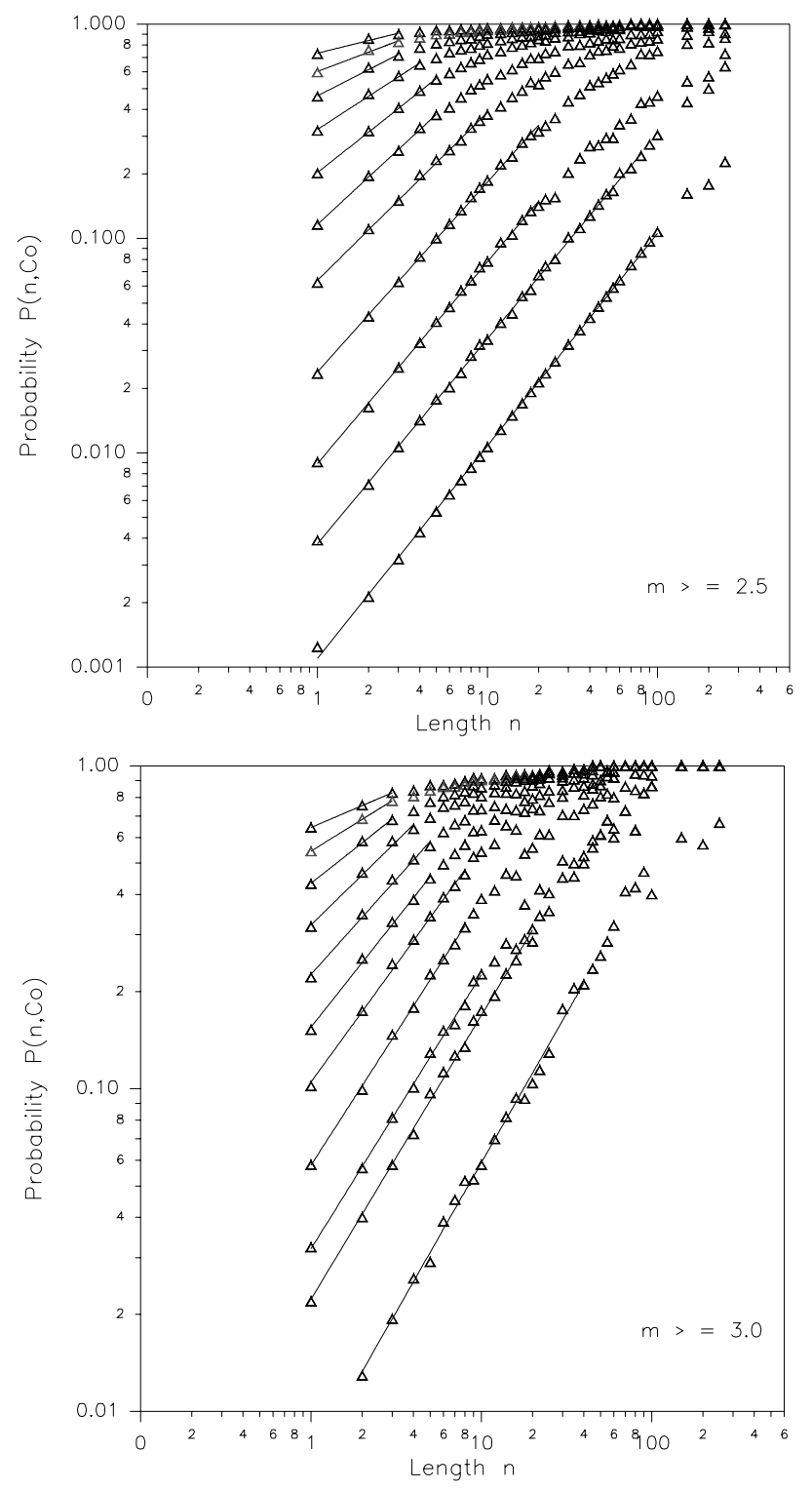

Fig. 2. Evolution of the probability $P\left(n, C_{0}\right)$ for series of distances. The cases of seismic events equalling or exceeding magnitudes of 2.5 and 3.0 are computed for 11 different threshold values of $C_{0}$ ranging from $50 \mathrm{~km}$ (top) to $550 \mathrm{~km}$ (bottom) at intervals of $50 \mathrm{~km}$.

can be found in Eckmann et al. (1986) and Stoop and Meier (1988). From a practical point of view, the Lyapunov exponents have to be computed for increasing values of $m$, up to observe their asymptotic evolution and to achieve the embedding dimension $d_{E}$.

It has to be taken into account that the values of all the introduced parameters could be strongly affected by a not high enough number of reconstructed vectors $Z_{i}$ From a more quantitatively point of view and according to Ruelle (1990), a meaningful correlation dimension $\mu$ should verify the relationship $\mu \leq 2 \ln (N)$, with $N$ being the number of reconstructed vectors. Whereas series of elapsed times and distances for the whole seismic catalogue are long enough to
Table 3. Hurst exponent, $H$, for the distance and elapsed time between consecutive events and square regression coefficients $\rho^{2}$. Distinction is made between four different threshold magnitudes of 2.5, 3.0, 3.5 and 4.0.

\begin{tabular}{cccccc}
\hline & Distance & \multicolumn{3}{c}{ Elapsed time } \\
\hline$m_{b}$ & $\rho^{2}$ & $H$ & $m_{b}$ & $\rho^{2}$ & $H$ \\
\hline 2.5 & 0.992 & 0.82 & 2.5 & 0.997 & 0.75 \\
3.0 & 0.993 & 0.80 & 3.0 & 0.997 & 0.75 \\
3.5 & 0.995 & 0.71 & 3.5 & 0.993 & 0.66 \\
4.0 & 0.980 & 0.62 & 4.0 & 0.975 & 0.65 \\
\hline
\end{tabular}

generate a high number $N$ of reconstructed vectors, those generated from seismic crises (aftershocks of a main event) are remarkably shorter and a high enough number of reconstructed vectors for the fulfilment of Ruelle's constraint is not assured. Consequently, analyses based on the reconstruction theorem are finally restricted to the whole seismic catalogue.

\section{Results}

With respect to the cluster analysis, we have to remember that only series of elapsed times and distances between consecutive seismic events for the whole catalogue and magnitudes greater than 2.5 and 3.0 are long enough to obtain reliable results. Table 2 summarises the cluster dimensions, $D$, obtained in each case. The square regression coefficients are always close to 1.0, as the example shown in Fig. 2 suggests, and summarised in Table 2. First, the relationship between $P\left(n, C_{0}\right)$ and $n$ departs systematically from a potential law for high values of $n$, or equivalently for $P\left(n, C_{0}\right)$ close to 1.0 , which is a quite common behaviour in some fractal analyses. Then, the clustering dimensions derived for the first threshold levels are doubtful, as they are obtained by only fitting a very few points. Second, the number of fitted points increases and dimensions are obtained with minor uncertainty for higher threshold levels. In short, seismic events associated with high elapsed times or distances tend to disperse within the seismic catalogue, diminishing their cluster dimension, in comparison with low and moderate threshold values of $C_{0}$. Third, large threshold levels are associated with cluster dimensions very close to 0 and a Poisson process, without fractal structure, could model the case of high elapsed times and long distances. In this way, the evolution of the clustering dimension would suggest an intensification of the fractal clustering with increasing values of the threshold value $C_{0}$, but constrained by the mentioned behaviour for large thresholds.

The re-scaled analysis is characterised by an almost perfect evolution of the maximum range and the standard deviation of the series in terms of a potential law and the square regression coefficients are in all cases very close to 1.0, as 
(a)

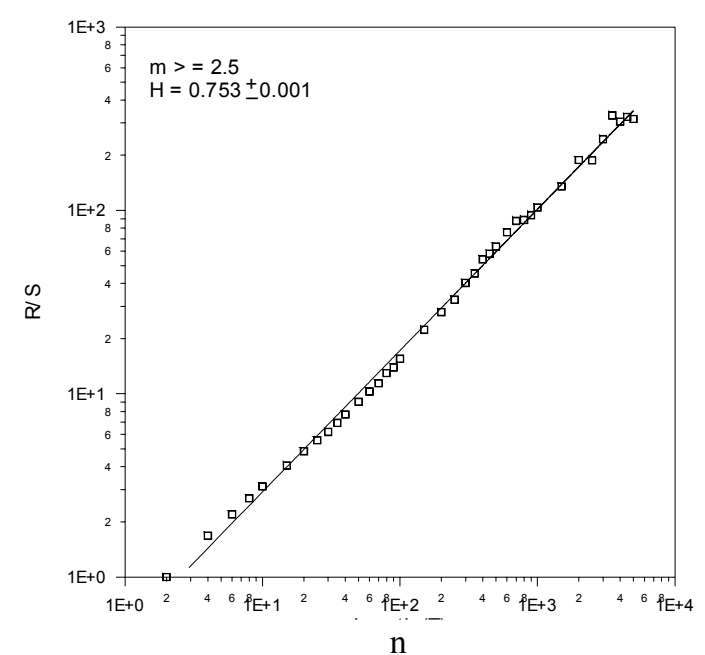

(b)

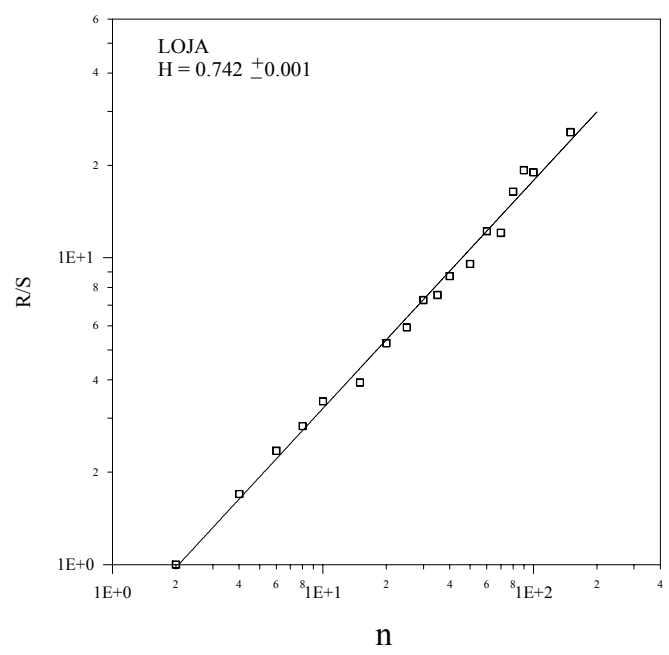

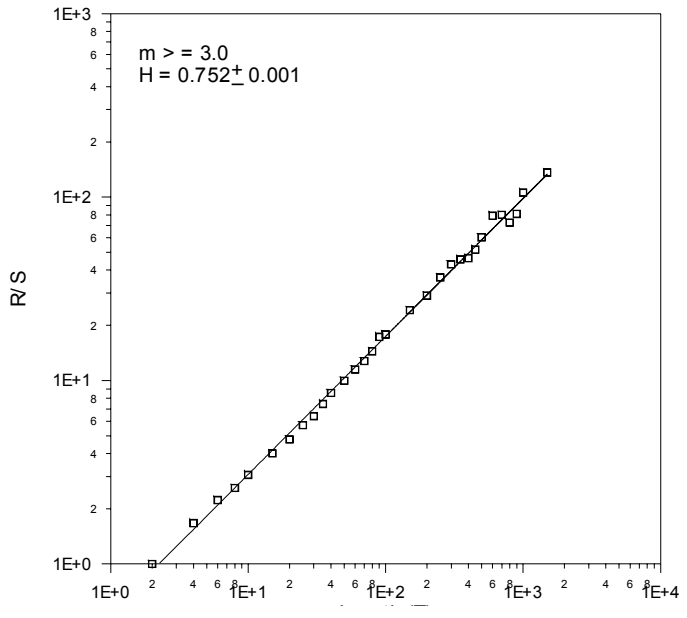

$\mathrm{n}$

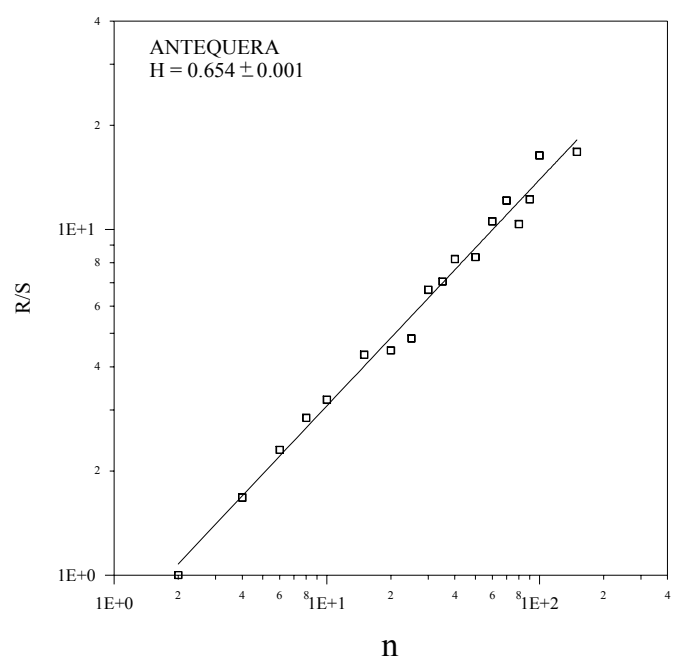

Fig. 3. Examples of re-scaled analysis for the elapsed time between consecutive events of the whole catalogue and threshold magnitudes of 2.5 and 3.0 (a) and for the distances and seismic crises of Loja and Antequera (b).

the two examples, shown in Fig. 3, illustrate. The set of Hurst parameters obtained both for the whole catalogue and for five seismic crises within the analysed area (Table 1) is summarised in Tables 3 and 4, where the square regression coefficients are included. According to Table 3 and the interpretation of the Hurst exponent, a tendency towards randomness could be assumed when the threshold magnitudes increase from 2.5 to 4.0 , both for the elapsed times and for the distances between consecutive events. The fact that the Hurst exponent decreases with the threshold magnitude, showing signs of a randomness, could be attributable to the relatively low number of events for $m_{b}$ greater than 3.5 and specially for those greater than 4.0. Nevertheless, the potential Eq. (2) performs very well empirical data, with square regression coefficients greater than 0.9. Consequently, the tendency to randomness is a feature to be considered. It is also remarkable that the mentioned behaviour should not be in agreement, at least with respect to elapsed times, with results derived by Bak et al. (2002), who obtained a universal behaviour for the waiting times of earthquakes recorded in California, with a simple unified scaling law from tens of seconds to tens of years. With respect to distances between events, it should be mentioned the work of Davidsen and Paczuski (2005), who deduced for the Southern California seismic activity that statistics of distances, ranging from 2 to $450 \mathrm{~km}$, are independent of the threshold magnitude as long as the catalogue is complete.

The results depicted in Table 4 suggest clear signs of timepersistence for the elapsed times derived from the seismic crisis of Adra, Agrón, Alborán and Loja, with Hurst exponents exceeding 0.79 and achieving the highest value of 0.87 for Loja. Nevertheless, the value of $H$ for Antequera is quite similar to those obtained for the series of distances derived for Alborán and Adra crisis, which suggest some signs of 
Table 4. Hurst exponent, $H$, for the series of distances and elapsed times derived from five seismic crisis within the area analysed. $\rho^{2}$ represents the square regression coefficient.

\begin{tabular}{|c|c|c|c|c|c|}
\hline Distance & & & Elapsed time & & \\
\hline Crisis & $H$ & $\rho^{2}$ & Crisis & $H$ & $\rho^{2}$ \\
\hline Adra & 0.62 & 0.989 & Adra & 0.81 & 0.995 \\
\hline $\operatorname{Adra}^{(1)}$ & 0.84 & 0.995 & Adra $^{(1)}$ & 0.85 & 0.997 \\
\hline Agrón & 0.78 & 0.993 & Agrón & 0.80 & 0.991 \\
\hline Agrón $^{(1)}$ & 0.82 & 0.997 & Agrón $^{(1)}$ & 0.85 & 0.994 \\
\hline Alborán & 0.66 & 0.992 & Alborán & 0.79 & 0.992 \\
\hline Alborán $^{(1)}$ & 0.83 & 0.993 & Alborán ${ }^{(1)}$ & 0.86 & 0.995 \\
\hline Antequera & 0.65 & 0.995 & Antequera & 0.68 & 0.993 \\
\hline Antequera $^{(1)}$ & 0.80 & 0.992 & Antequera $^{(1)}$ & 0.86 & 0.988 \\
\hline Loja & 0.74 & 0.996 & Loja & 0.87 & 0.993 \\
\hline $\operatorname{Loja}^{(1)}$ & 0.77 & 0.995 & $\operatorname{Loja}^{(1)}$ & 0.81 & 0.998 \\
\hline
\end{tabular}

Table 5. Embedding dimension, $d_{E}$, Kolmogorov entropy, $K$, correlation dimension, $\mu$, and the two first positive Lyapunov exponents, $\lambda_{1}$ and $\lambda_{2}$, for threshold magnitudes of 2.5 and 3.0.

\begin{tabular}{cccccc}
\hline \multicolumn{2}{c}{$D(\mathrm{~km})$} & \multicolumn{3}{c}{$T$ (days) } \\
\hline$m_{b}$ & 2.5 & 3.0 & $m$ & 2.5 & 3.0 \\
$d_{E}$ & 15 & 15 & $d_{E}$ & 14 & 14 \\
$K$ & $0.330 \pm 0.021$ & $0.215 \pm 0.009$ & $K$ & $0.330 \pm 0.009$ & $0.232 \pm 0.006$ \\
$\mu$ & 5.8 & 3.8 & $\mu$ & 4.1 & 2.4 \\
$\lambda_{I}$ & $0.143 \pm 0.003$ & $0.167 \pm 0.001$ & $\lambda_{I}$ & $0.174 \pm 0.004$ & $0.178 \pm 0.002$ \\
$\lambda_{2}$ & $0.105 \pm 0.002$ & $0.115 \pm 0.001$ & $\lambda_{2}$ & $0.121 \pm 0.001$ & $0.123 \pm 0.004$ \\
\hline
\end{tabular}

randomness. The other three crises, Antequera, Loja and specially Agrón, have a behaviour closer to persistence. Another relevant feature is the comparison between Hurst exponents derived for every seismic crisis and those derived taking a subset of the whole catalogue, spatially coincident with the geographical area of every crisis but extending in time to the entire observational period (1985-2000). Whatever the series or area of crisis considered, it is worth mentioning that the option covering all the recording period is always characterised by an enhancement of persistence in front of randomness. Two relevant examples are those of Adra and Alborán, for which $H$ increases $25-35 \%$ when the entire recording period of the catalogue is considered.

Both cluster dimensions and Hurst exponents have been obtained with a high degree of accuracy, in spite of the series of elapsed times and distances are not very long in some cases (Table 1). Square regression coefficient achieves values exceeding 0.97, fact that suggests an evolution in terms of potential laws. An illustrative example of the accuracy obtained is illustrated by Figs. $3 \mathrm{a}$ and $3 \mathrm{~b}$, where the square regression coefficients are very close to 1.0 and the uncertainties on $H$ are as much as \pm 0.001 .

With respect to the fractal analysis, based on the reconstruction theorem, a question concerning the parameter $q$ used in the generation of reconstructed vectors have to be
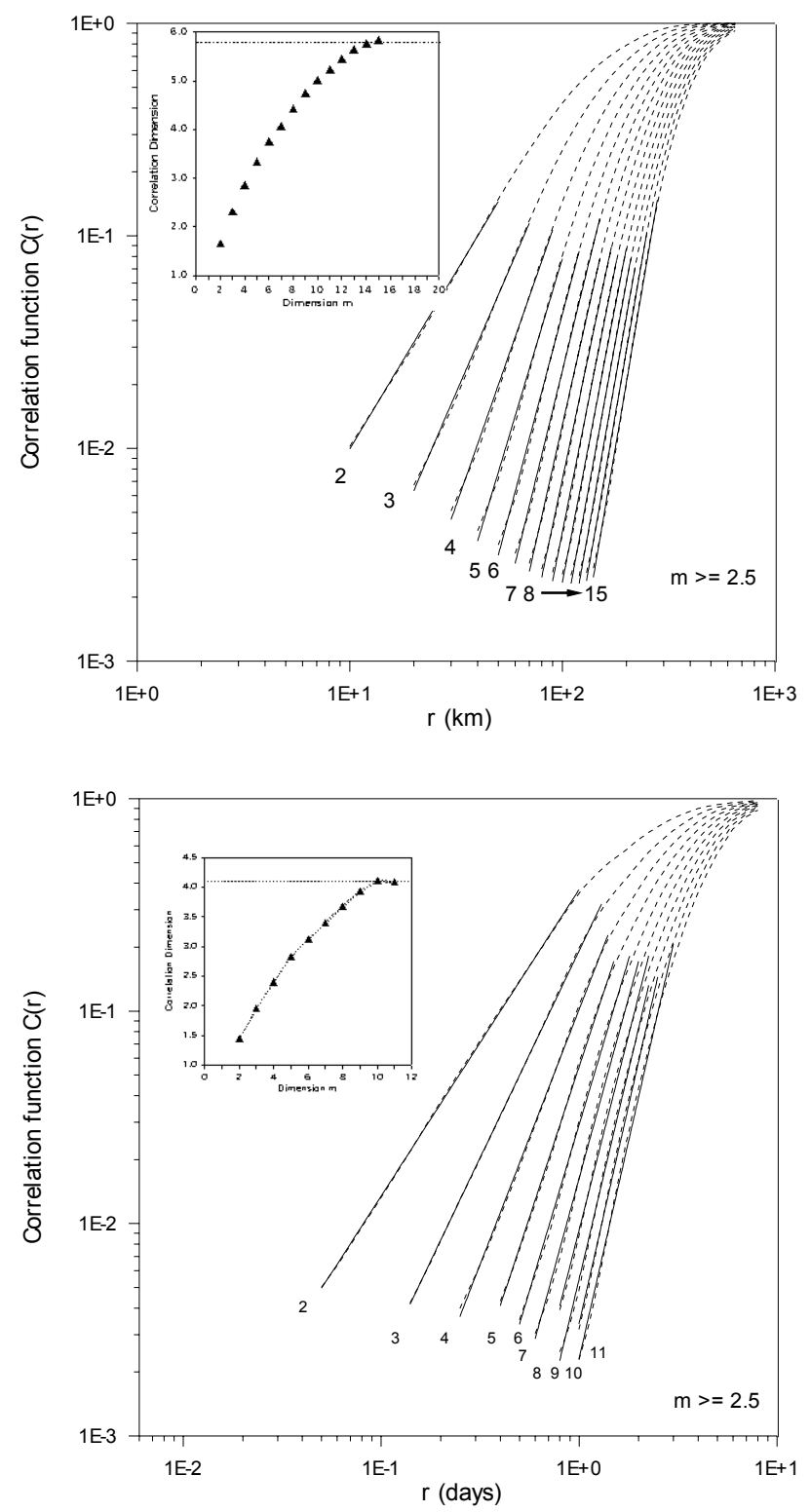

Fig. 4. Correlation function and correlation dimension (inner rectangle) for series of distances and elapsed times between consecutive events. Reconstruction dimensions from 2 to 15 and from 2 to 11 are tested, respectively.

clarified. The correlation dimension and the Kolmogorov entropy of a reconstructed system are independent of $q$, except for finite time series (Diks, 1999). Wu (1995) and Correig et al. (1997), propose that a good approach of this parameter should be given by the lag order of the first zero-crossing of the autocorrelation function of the series analysed. Alternatively, Diks (1999) and Buzug and Pfister (1992) assume that a small value of $\mathrm{q}$ can be used if high embedding dimensions are finally achieved. In fact, the time window $q(m-1)$ seems to be the relevant factor of the reconstruction.

This last analysis, for the whole seismic catalogue, is summarised in Table 5. Relevant differences are not observed between the series of distances and elapsed times, at least 
with respect to Lyapunov exponents, Kolmogorov entropy and embedding dimension. Consequently the "loss of memory" of the dynamical mechanism governing the seismicity and the instability of a predictive algorithm should be quite similar. Additionally, the relatively high value of $d_{E}$ manifests signs of randomness for both the spatial and temporal evolution of the seismicity. Nevertheless, the correlation dimension suggests some differences in the complexity of the non-linear equations describing the dynamical mechanism.

A relevant question is to chose the right interval of $r$ for a wright computation of the parameter $\mu$. The power law given by Eq. (5) is many times disturbed by a set of effects, extensively discussed by Theiller $(1988,1990)$, and $\log \{C(r)\}$ behaves linearly with $\log (r)$ for only a range of $r$. As Correig and Urquizú (1996) and Diks (1999) propose, a solution consists of choosing the interval of $r$ for which the derivative of $\log [C(r)]$ with respect $\log [r]$ is approximately constant. This procedure is equivalent to a visual inspection of curves $C(r)$, detection of the linear range in a log-log scale and computation of dimension $\mu$ by means of a standard linear regression. Unfortunately, this process is difficult to implement in computational routines and it requires a laborious treatment. Figure 4 depicts an example of correlation integral and the corresponding dimension for events exceeding or equalling to the threshold magnitude of 2.5. The linear evolution of the correlation integral can be clearly observed in a log-log scale for a relatively wide range of the argument $r$. Nevertheless, a set of factors and shortcomings such as lacunarity, sampling interval, random components or finite length of the series (Theiler, 1988, 1990; Diks, 1999) leads to relevant changes in the upper and lower limits for which the correlation dimension can be accurately computed when several reconstruction dimensions $m$ are attempted. In spite of all these shortcomings, the evolution towards an asymptotic value of the correlation dimension for a high enough dimension $m$ is observed. Figure 5 shows the evolution with the reconstruction dimension $m$ of the Kolmogorov entropy towards asymptotic values for the distances between consecutive events and threshold magnitude of 2.5 and 3.0. According to the interpretation of $K$, the set of events exceeding or equalling to 3.0 depicts minor "loss of memory" and chaotic behaviour in comparison with those defined by the threshold level of 2.5.

Finally, Fig. 6 describes the evolution of the first and second Lyapunov exponents with the reconstruction dimension $\mathrm{m}$ for seismic events with magnitudes exceeding or equalling to 2.5 and 3.0, respectively, both for series of distances and of elapsed times between consecutive seismic events. Even though some differences are detected in the four cases, they are not very relevant and the instability of a predictive mechanism of the seismicity should be assumed quite similar in all cases.

A question that could be debatable is the estimation of the correlation dimension, defined as the asymptotic value achieved for a high enough embedding dimension. This dimension $d_{E}$, and the associated correlation dimension $\mu$, could be assumed well estimated due to the agreement with

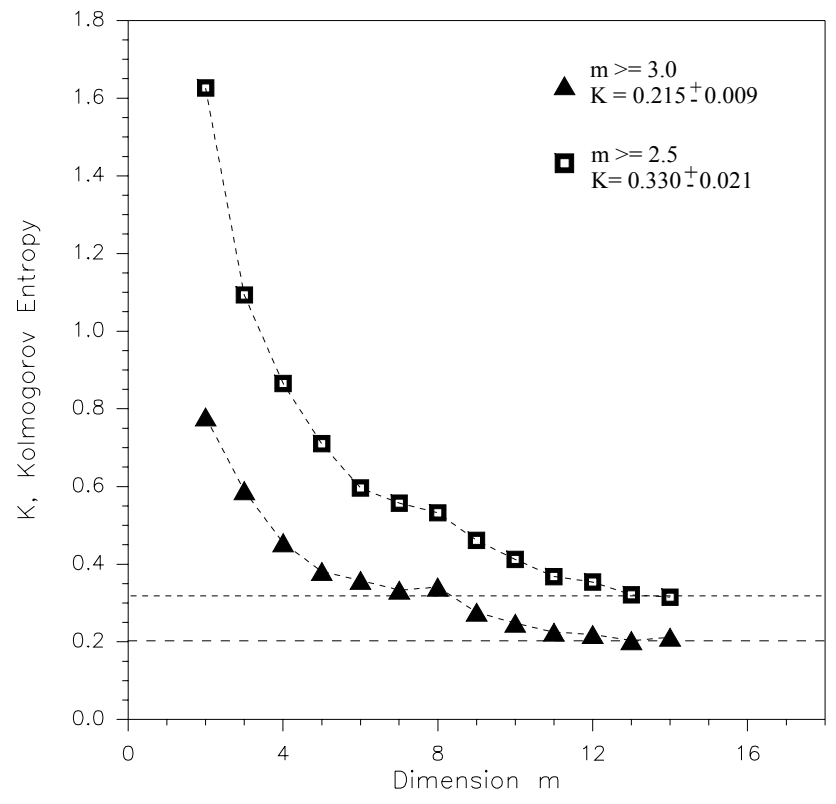

Fig. 5. Asymptotic values achieved by the entropy after a high enough dimension $\mathrm{m}$ for the series of distances between consecutive events and threshold magnitudes of 2.5 and 3.0.

results derived from the evolution of the Kolmogorov entropy and the Lyapunov exponents with dimension $m$. In spite of a slightly higher embedding dimension should not be discarded, examples shown in Figs. 4, 5, and 6 suggest that changes in entropy and Lyapunov exponents would be minima and consequences concerning loss of memory, unstable prediction and number of nonlinear equations describing the dynamical system will be very similar.

\section{Conclusions}

The set of fractal analyses proposed (cluster dimension, Hurst exponent, correlation dimension, Kolmogorov entropy and Lyapunov exponents) manifests a fractal behaviour of the seismic activity in the Southern Iberian Peninsula. The dissipative dynamical system that should describe the seismic activity in the Southern Iberian Peninsula is a nonlinear underlying mechanism, with a relatively high number of equations depending on the series and threshold magnitude considered. The present objective is not to propose a system of nonlinear equations to design a predictive schema for the seismicity of the Southern Iberian Peninsula, but investigate different behaviours for several thresholds levels, both for elapsed times and distances. The prediction of the dynamical system, with signs of chaotic and dissipative behaviour due to the Kolmogorov entropies computed, seems to be clearly unstable due to the presence of outstanding positive Lyapunov exponents for all series and threshold magnitudes. Nevertheless, these characteristics do not change remarkably either with the series considered or with the threshold magnitude. Only the evolution of the Hurst exponent with the 

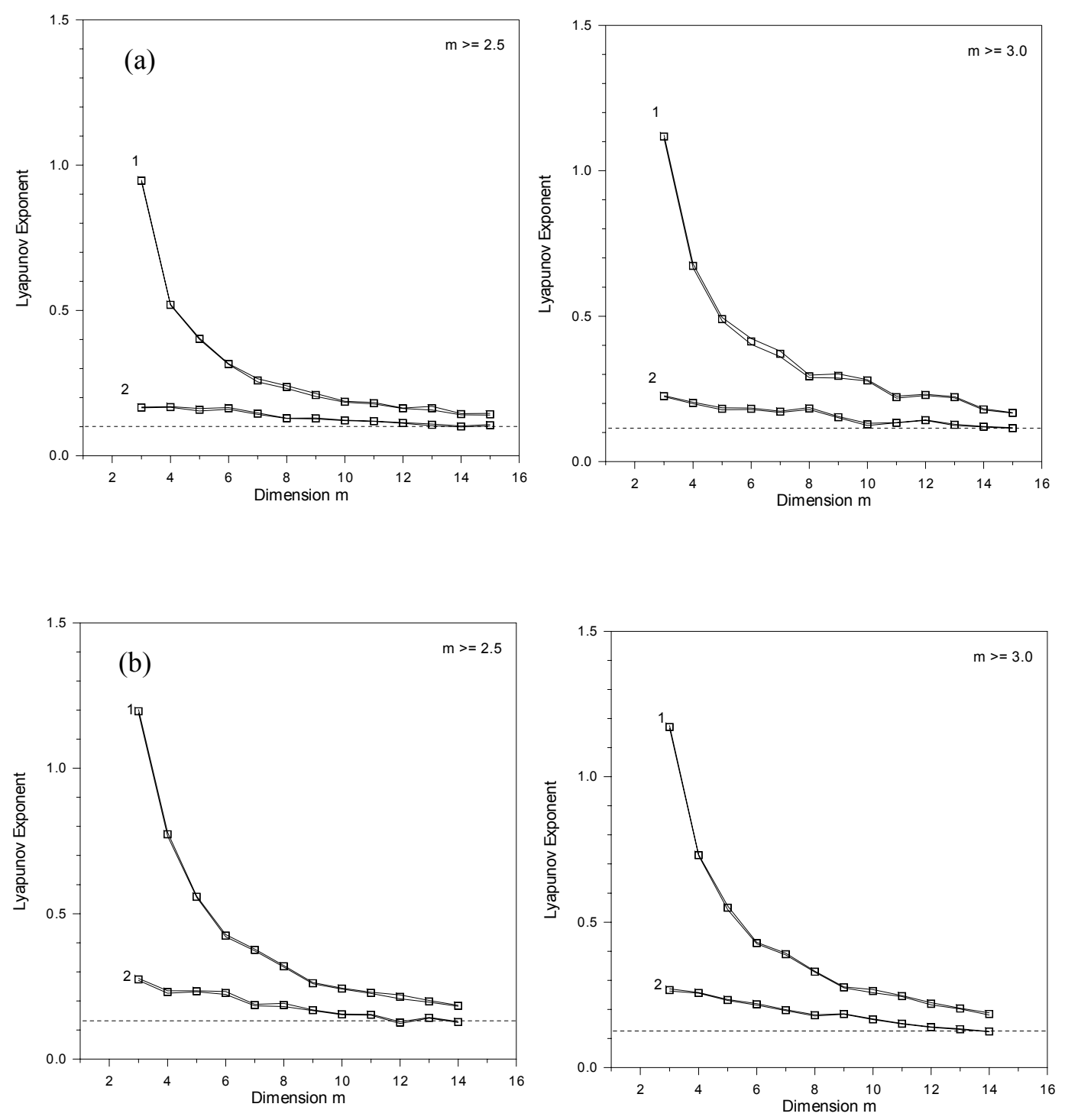

Fig. 6. Evolution of the first and second Lyapunov exponents with the reconstruction dimension $m$ for seismic events with magnitudes equalling or exceeding 2.5 and 3.0 for (a) series of distances and (b) series of elapsed times between consecutive seismic events.

increasing magnitude suggests that the use of just high magnitudes does not simplify the predictive problem. On the contrary, it would become more complex and with outstanding randomness. Moreover, the relatively large embedding dimension, for which values of entropy, correlation dimension and Lyapunov exponents saturate, suggests the presence of a relevant randomness in the dynamical mechanism. This randomness, of course, increases the difficulties for earthquake prediction. These clear signs of randomness, chaotic behaviour and unstable prediction of future states of seismicity have been detected in other seismotectonic areas of the Earth such as the Antarctica (Correig et al., 1997). Nevertheless, the predictive instability of the dynamical dissipative system governing the seismicity is lesser in our case, as suggested by a comparison of our Lyapunov exponents and those derived by Correig et al. (1997).
The five seismic crises, which belong to the whole seismic catalogue analysed, do not generate long enough series to apply the complete fractal analysis proposed for the entire catalogue. Nevertheless, a close look at Hurst exponents leads to some interesting conclusions. Although the minimum magnitude for most of the seismic crisis is lower than the minimum well recorded magnitude of 2.5 for the entire catalogue, due to the close monitoring of these crises, Hurst exponents are similar to those derived for threshold magnitudes of 3.5 and 4.0, specially for the series of distances between events. A certain degree of randomness, generated in both cases by some loss on information concerning the seismic activity, can be assumed. Magnitudes less than 2.5 have been discarded in the first case and time length and space coverage of a seismic crisis are considerably lesser than those offered by the whole catalogue. Additionally, it can not be absolutely 
guaranteed that all aftershocks exceeding the threshold magnitude have been detected by the seismic arrays. Another question to mention is that Hurst exponents depart significantly from randomness towards time-persistence when subsets of the whole catalogue, covering the areas of every seismic crisis, but expanding in time to the whole observational period, are considered. Completeness of the catalogue, interpreted as appropriate time expand and spatial coverage, and appropriate magnitude range, seem to be very important factors for the design of successful predictive algorithms.

Finally, a joint analysis of elapsed times and distances could be another interesting point of view for a better understanding of the dynamical mechanism of the seismicity. A first possibility could be based on a Lévy flight model (Vázquez et al., 1998; Posadas et al., 2002). Alternatively, Tosi et al. (2004) introduced a space-time combined correlation integral and proposed time and space correlation dimensions with very similar definitions to that used at present.

Acknowledgements. This research was supported by the Spanish Ministry of Science and Technology through the projects REN2001-2418-C04-01 RIES and REN2003-07170 RIES.

Edited by: J. Davidson

Reviewed by: two referees

\section{References}

Bak, P., Christensen, K., Danon, L., and Scanlon, T.: Unified scaling law for earthquakes, Phys. Rev. Lett., 88, 12, 178501, 1-4, 2002.

Buzug, T. and Pfister, G.: Comparison of algorithms calculating optimal parameters for delay time coordinates, Physica D, 58, 127, 1992.

Cohen, A. and Procaccia, I.: Estimation of the Kolmogorov entropy from time signals of dissipative and conservative dynamical systems, Phys. Rew. (A), 31, 1872-1882, 1983.

Correig, A. M. and Urquizú, M.: Chaotic behaviour of coda waves in the Eastern Pyrenees, Geophys. J. Int., 126, 113-122, 1996.

Correig, A. M., Urquizú, M., and Vila, J.: Aftershock series of event February 18, 1996: an interpretation in terms of self-organised criticality, J. Geophys. Res., 102, B12, 27 407-27 420, 1997 a.

Correig, A. M., Urquizú, M., Vila, J., and Martí, J.: Analysis of the temporal occurrence of seismicity at Deception Island (Antarctica), A non-linear approach, Pure Appl. Geophys., 149, 553574, 1997b.
Davidsen, J. and Paczuski, M.: Analysis of the spatial distribution between successive earthquakes, Phys. Rev. Lett., 94, 048501, 2005.

Diks, C.: Nonlinear time series analysis, Nonlinear Time Series and Chaos (4), World Scientific, London, 209pp, 1999.

Eckmann, J. P., Oliffson, S., Ruelle, D., and Ciliberto, S.: Lyapunov exponents from time series, Phys.. Rew. (A), 34, 6, 4971-4979, 1986.

Feder, J.: Fractals, Ed. Plenum, New York, 1988.

Goltz, C.: Fractal and Chaotic Properties of Earthquakes, Lecture Notes in Earth Sciences (77), Springer, Berlin, 178pp, 1997

Grassberger, P. and Procaccia, I.: Characterization of strange attractors, Phys. Rev. Lett., 50, 346-349, 1983a.

Grassberger, P., and Procaccia, I.: Estimation of the Kolmogorov entropy from a chaotic signal, Phys. Rev. (A), 28, 2591, 1983b.

Korvin, G.: Fractal models in Earth sciences, Elsevier, Amsterdam, 1992.

Posadas, A. M., Morales, J., Vidal, F., Sotolongo-Costa, O., and Antoranz, J. C.: Continuous Time Random Walks and Seismic Series: Southern Spain Seismic Series and Lévy Flights, J. Seismol., 6, 62-67, 2002.

Ruelle, D.: Deterministic chaos: The Science and the Fiction, Proc. R. Soc. London A, 427, 241-248, 1990.

Smalley, R. F., Chatelain, J. L., Turcotte, D. L., and Prébot, R.: A fractal approach to the clustering of earthquakes: application to the seismicity of New Hebrides, Bull. Seism. Soc. Am., 77, 4, 1368-1381, 1987.

Stoop, R. and Meier, P. F.: Evaluation of Lyapunov exponents and scaling functions from time series, J. Opt. Soc. Am. (B), 5, 10371045, 1988.

Takens, F.: Detecting strange attractors in turbulence. In: Lecture Note in Mathematics, edited by: Rand, D. A. and Young L. S., Springer, Berlin, 1981.

Theiler, J.: Lacunarity in a best estimator of fractal dimension, Phys. Lett. (A), 133, 195-200, 1988

Theiler, J.: Estimating fractal dimension, J. Opt. Soc. Am., 7, 1055$1073,1990$.

Tosi, P., de Ruveis, V., Loreto, V., and Pietronero, L.: Influence length and space-time correlation between earthquakes, Physics, 0409033, http://arxiv.org/abs/physics/0409033, 2004.

Turcotte, D. L.: Fractal and Chaos in Geology and Geophysics, Cambridge University Press, New York, 1992.

Urquizú, M. and Correig, A. M.: Analysis of seismic dynamical systems, J. Seismol., 2, 159-171, 1998.

Vázquez, A., Sotolongo-Costa, O., and Brouers, F.: Diffusion regimes in Lévy flights with trapping, Physica A, 264, 424-431, 1998.

Wu, Z.: Remark on metric analysis of recontrucyerd dynamics from chaotic time series, Physica D, 85, 485-495, 1995. 